\title{
Atypical bronchoplasty to preserve the lung parenchyma: The bronchofolding technique
}

\author{
Mitsuhiro Kamiyoshihara, MD, PhD, ${ }^{a}$ Takashi Ibe, MD, ${ }^{a}$ Atsushi Takise, MD, PhD, and Izumi Takeyoshi, MD, PhD, ${ }^{\mathrm{c}}$ \\ Gunma, Japan
}

M any cases involving atypical bronchoplasty have been reported. ${ }^{1-3}$ The patient reported here presented with an elongated oval bronchial defect involving the bronchus intermedius. To preserve the lung parenchyma, we performed a wedge resection of the remaining wall between the upper and middle bronchus and anastomosed the bronchial stumps. We consider this procedure as a type of wedge or flap lobectomy, as described in Khargi and colleagues ${ }^{1}$; that is, the bronchial stump was anastomosed by folding the bronchial wall of the bronchus intermedius. Here we describe a successful procedure for an atypical bronchoplasty using the bronchofolding technique.

\section{Clinical Summary}

A 72-year-old man was referred to our hospital for further investigation of an abnormal shadow seen on a chest radiograph taken during a medical check-up. Computed tomographic (CT) analysis revealed the shadow of a mass, measuring $3 \times 2 \mathrm{~cm}$, that originated in the superior segment (S6) of the right lower lobe (Figures 1, A, and 2, $A$ ). Flexible bronchoscopy showed no endobronchial tumor, but it did show stenosis of the bronchus intermedius, where it was compressed by the mass. Magnetic resonance imaging of the brain and CT scanning of the abdomen uncovered no evidence of distant metastasis. We suspected primary lung cancer, classified as T3 N2 M0, stage IIIA disease, and scheduled a radical operation.

The chest was entered through the fifth intercostal space with a lateral thoracotomy after achievement of general anesthesia with a double-lumen endotracheal tube. A needle aspiration biopsy of the tumor yielded a diagnosis of squamous cell carcinoma of the lung. The tumor originating in S6 had invaded the outer wall of the intermediate trunk of the bronchus. The tumor involved the azygous vein and its posterior portion of the arch and the posterior mediastinal pleura. After dividing the azygous vein, vagus nerve, and posterior mediastinal pleura, the pulmonary artery branch to the lower lobe and inferior pulmonary vein was divided by using a me-

From the Departments of General Thoracic Surgery ${ }^{\mathrm{a}}$ and Respiratory Medicine, ${ }^{\mathrm{b}}$ Maebashi Red Cross Hospital, and the Division of Thoracic and Visceral Organ Surgery, ${ }^{\mathrm{c}}$ Gunma Graduate University School of Medicine, Maebashi, Gunma, Japan.

Received for publication Sept 17, 2007; revisions received Oct 26, 2007; accepted for publication Dec 27, 2007.

Address for reprints: Mitsuhiro Kamiyoshihara, MD, PhD, Department of General Thoracic Surgery, Maebashi Red Cross Hospital, 3-21-36 AsahiCho, Maebashi, Gunma 371-0014, Japan (E-mail: micha2005jp@yahoo.co. jp).

J Thorac Cardiovasc Surg 2008;135:1184-5

$0022-5223 / \$ 34.00$

Copyright $\odot 2008$ by The American Association for Thoracic Surgery doi:10.1016/j.jtcvs.2007.12.031 chanical stapler. The tumor was extirpated with division of the lower bronchus, the base of which was cut out in an elongated oval (Figures $1, B$, and 2, B). Then the bronchial stump was confirmed by means of frozen sectioning to be pathologically free of cancer.

There was a large defect in the wall of the intermedius trunk, including the lower lobe bronchial stump. We resected a wedge of the bronchial wall (Figures 1, $C$, and 2, $C$ ) and anastomosed the bronchial stumps while folding the middle bronchus superiorly with interrupted sutures of absorbable 3-0 monofilament. The anastomosis of the bronchus was completed as shown (Figure 1,D). Finally, absorbable sealing materials were put around the bronchial anastomosis. Mediastinal and hilar lymph node dissection was added, and the operation was finished.

Pathologically, the patient was given a diagnosis of primary squamous cell carcinoma, which was classified as T3 N0 M0 disease, stage IIB. The postoperative course was uneventful. A postoperative 3-dimensional CT scan showed no stenosis and no recurrence at the anastomosis (Figure 2, D). The patient was doing well without local recurrence 6 months after the operation and is being followed as an outpatient.

\section{Discussion}

It was obvious that directly closing the stump of the lower bronchus was impossible because of the large defect in the bronchus. Initially, we considered a bilobectomy, but we wanted to preserve the healthy lung, if possible. Therefore we considered a lower sleeve lobectomy with an anastomosis between the intermediate and middle bronchus using the telescoping anastomosis technique. That was reasonable and proper for a caliber mismatch. ${ }^{4}$ However, there was a sufficient tumor-free region of the intermediate bronchus. Also, if even a small amount of tissue was left, and then we could decrease the risk of anastomotic insufficiency because the tension between the bronchial stumps decreased. Therefore we developed a bronchoplasty that cut the bronchial wall obliquely to the cartilaginous rings and folded the bronchus. We usually cut the bronchus parallel to the cartilaginous rings, and obliquely cutting the bronchus made us concerned with anastomotic insufficiency. After much cogitation, we decided to adopt the presented technique in the motif of a slide tracheoplasty, as reported by Grillo and coworkers. 5

For an elongated oval defect of the bronchus intermedius, we propose using the procedure presented here. By adding a wedge resection for the part remaining between the upper and middle bronchus, we can adjust the discrepancy and decrease the tension between the bronchial stumps. We consider our procedure to be a combination of wedge and flap lobectomies and call this original technique the bronchofolding technique. 

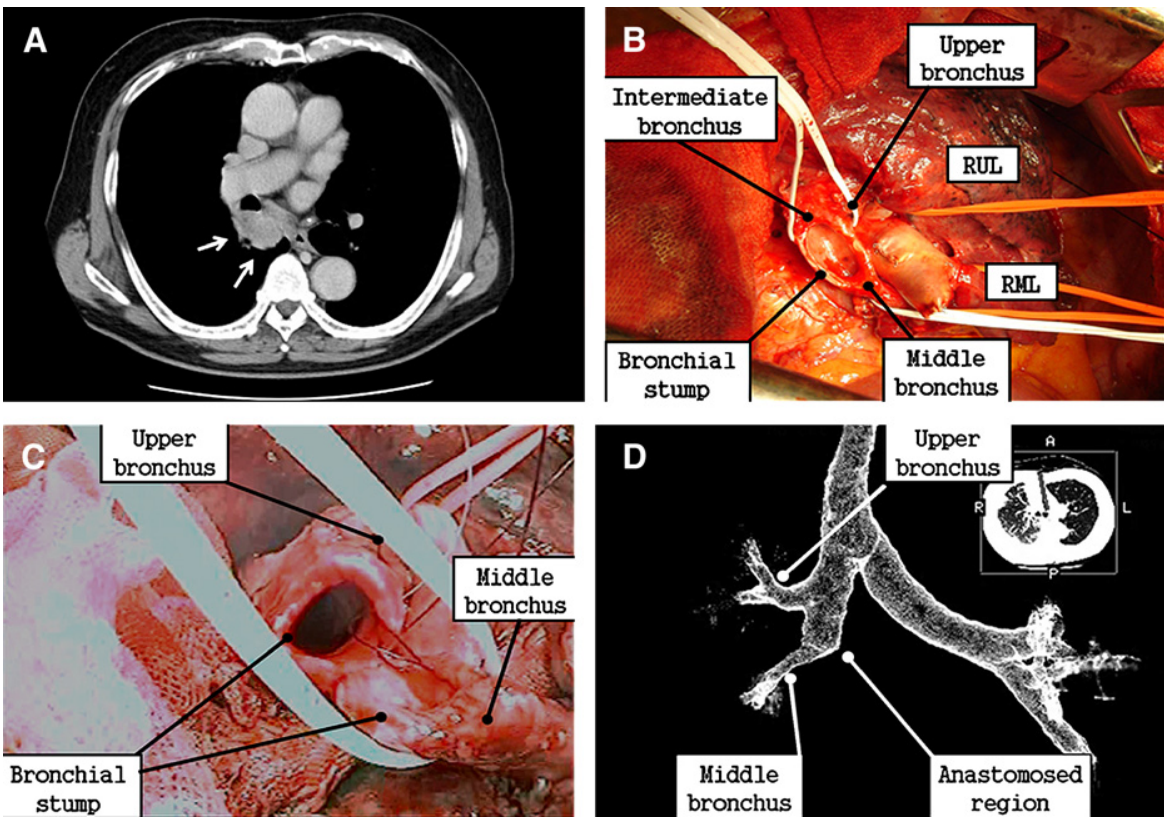

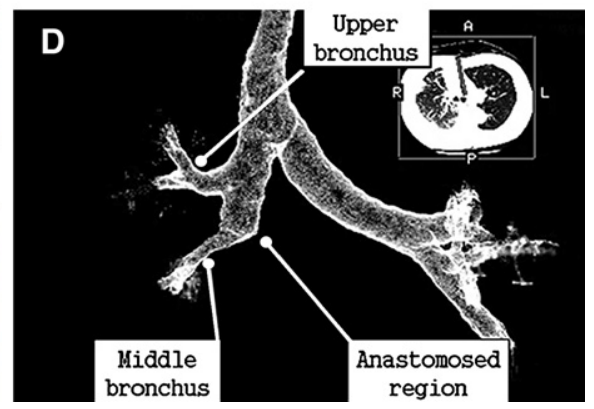

Figure 1. A, Computed tomographic analysis reveals the shadow of a mass, measuring $3 \times 2 \mathrm{~cm}$, that originates in the superior segment (S6) of the right lower lobe. $B$, The tumor was extirpated with division of the lower bronchus, the base of which was cut out in an elongated oval. RUL, Right upper lobe; RML, right middle lobe. C, We resected a wedge of the bronchial wall. D, Interrupted sutures of absorbable 30 monofilament were used. The anastomosis of the bronchus was completed as shown.
A

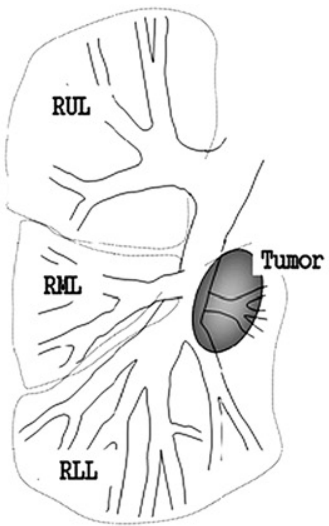

Anterior View of the right lung

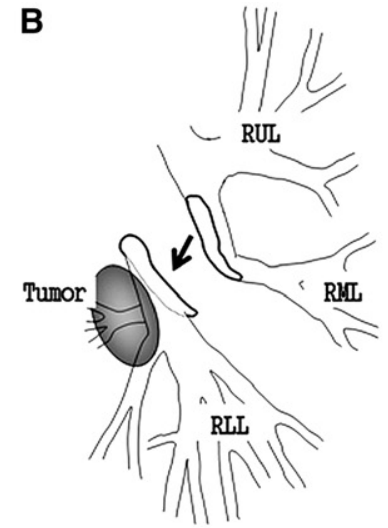

Posterior View of the right lung
C

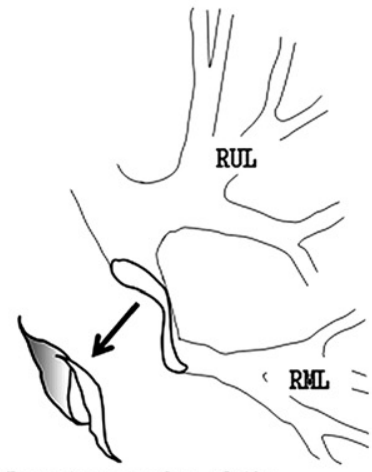

Renoving a wedge of the bronchial wall
D

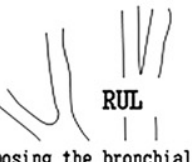

Anastomosing the bronchial stumps with folding the midlle bronchus superiorly

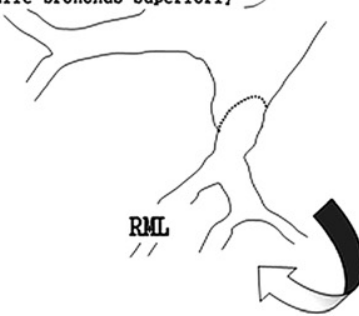

Anterior View of the right lung

Figure 2. A-C, Diagrams of the surgical procedure corresponding to Fig 1, A, B, and C, respectively. D, Postoperative 3-dimensional CT scan showing no stenosis at the anastomosis. $R U L$, Right upper lobe; $R M L$, right middle lobe; $R L L$, right lower lobe.

\section{References}

1. Khargi K, Duurkens VA, Versteegh MM, Huysmans HA, Quanjer PH, Verzijlbergen FF, et al. Pulmonary function and postoperative complications after wedge and flap reconstructions of the main bronchus. J Thorac Cardiovasc Surg. 1996;112:117-23.

2. Okada M, Tsubota N, Yoshimura M, Miyamoto Y, Matsuoka H, Satake S, et al. Extended sleeve lobectomy for lung cancer: the avoidance of pneumonectomy. J Thorac Cardiovasc Surg. 1999;118:710-3.

3. Kotoulas C, Lazopoulos G, Foroulis C, Konstantinou M, Tomos P, Lioulias A. Wedge resection of the bronchus: an alternative bronchoplas- tic technique for preservation of lung tissue. Eur J Cardiothorac Surg. 2001;20:679-83.

4. Hollaus PH, Janakiev D, Pridun NS. Telescope anastomosis in bronchial sleeve resections with high-caliber mismatch. Ann Thorac Surg. 2001;72: $357-61$.

5. Grillo HC, Wright CD, Vlahakes GJ, MacGillivray TE. Management of congenital tracheal stenosis by means of slide tracheoplasty or resection and reconstruction, with long-term follow-up of growth after slide tracheoplasty. J Thorac Cardiovasc Surg. 2002;123: $145-52$. 